\title{
Predicting Binding Sites of Hepatitis C Virus Complexes Using Residue Binding Propensity and Sequence Entropy
}

\author{
Guang-Zheng Zhang^, Chirag Nepal, and Kyungsook Han ${ }^{\star \star}$ \\ School of Computer Science \& Engineering, Inha Univ., Incheon 402751, Korea \\ aokunzhang@126.com, chirag@inhaian.net, khan@inha.ac.kr
}

\begin{abstract}
Hepatitis $\mathrm{C}$ virus (HCV) remains a dangerous health problem for the reason that the mechanism of hepatocyte infection is still unknown. Hence, much attention has been put on the problem of interaction between $\mathrm{HCV}$ and human proteins. However, the research is still standing at the beginning point due to the lack of structure information of $\mathrm{HCV}$ and human proteins. We extracted the most representative set of 18 complexes all known HCV protein complexes involving human proteins, and computed the binding propensity of each residue and sequence entropy of each HCV protein. analyzed the most representative set of 18 complexes. Using a radial basis function neural network (RBFNN), we predicted binding sites with an overall sensitivity of $77 \%$. The approach will help understand the interaction between $\mathrm{HCV}$ and human proteins.
\end{abstract}

Keywords: hepatitis C virus, radial basis function neural networks, binding site prediction, binding propensity, sequence entropy.

\section{Introduction}

Since the discovery of hepatitis $\mathrm{C}$ virus (HCV) in 1986, much attention has been focused on the research of interactions between $\mathrm{HCV}$ and human proteins. However, identifying the patterns of interactions between these proteins is hindered by a lack of structure information on these proteins. Hence, identification of the binding sites in HCV proteins, one of the most important median steps towards the interaction between $\mathrm{HCV}$ and human complex, has been developed for the purpose of understanding the HCV interaction mechanism [1].

However, the exact location of the binding sites within complexes, which can not always be addressed with X-ray crystallography, NMR or other experimental approaches, poses a problem. As an extension of our previous work [2, we have developed a radial basis function neural network (RBFNN) model for predicting the binding sites of HCV complexes. The RBFNN model is used to capture the relations between $\mathrm{HCV}$ primary sequence features and the known binding sites.

\footnotetext{
* This work is done while G.-Z. Zhang is on leave from the Red Star Institution, Hefei 230031, Anhui, China.

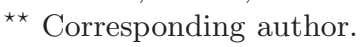


Table 1. Dataset of non-redundant HCV complexes with multiple chains

\begin{tabular}{|c|c|c|c|c|}
\hline $\begin{array}{l}\text { PDB } \\
\text { code }\end{array}$ & Chains & Description & $\begin{array}{r}\text { Sequence } \\
\text { length }\end{array}$ & $\begin{array}{r}{ }^{\natural} \text { Number of } \\
\text { known binding } \\
\text { residues }\end{array}$ \\
\hline $1 \mathrm{CSJ}$ & $\mathrm{A} / \mathrm{B}$ & Crystal str. of the RNA polymerase of HCV & $531 / 531$ & $32 / 33$ \\
\hline $1 \mathrm{NHU}$ & $\mathrm{A} / \mathrm{B}$ & HCV RNA Polymerase complex with analogue inhibitor & $578 / 578$ & $12 / 12$ \\
\hline${ }^{b} 1 \mathrm{~A} 1 \mathrm{R}$ & $\begin{array}{l}\mathrm{A} / \mathrm{B} \\
\mathrm{C} / \mathrm{D}\end{array}$ & HCV NS3 protease domain: NS4A peptide complex & $\begin{array}{r}198 / 198 \\
23 / 23\end{array}$ & $43 / 53$ \\
\hline $1 \mathrm{C} 2 \mathrm{P}$ & $\mathrm{A} / \mathrm{B}$ & Hepatitis C virus NS5B RNA-Dependent ... & $576 / 576$ & $14 / 14$ \\
\hline 1HEI & $\mathrm{A} / \mathrm{B}$ & Str. of the hepatitis $\mathrm{C}$ virus RNA Helicase domain & $451 / 451$ & $38 / 196$ \\
\hline${ }^{b} 1 \mathrm{~N} 64$ & $\begin{array}{r}\mathrm{H} / \mathrm{L} \\
\mathrm{P}\end{array}$ & Immunodominant antigenic site on $\mathrm{HCV}$ protein ... & $\begin{array}{r}220 / 218 \\
16\end{array}$ & $56 / 117$ \\
\hline $1 \mathrm{NHV}$ & $\mathrm{A} / \mathrm{B}$ & HCV RNA Polymerase in complex with ... & $578 / 578$ & $12 / 12$ \\
\hline $1 \mathrm{ZH} 1$ & $\mathrm{~A} / \mathrm{B}$ & Hepatitis C virus NS5B RNA-Dependent genotype 2a & $163 / 163$ & $24 / 19$ \\
\hline 2AWZ & $\mathrm{A} / \mathrm{B}$ & HCV NS5b RNA Polymerase in complex inhibitor (5h) & $580 / 580$ & $20 / 17$ \\
\hline $2 \mathrm{AX} 1$ & $\mathrm{~A} / \mathrm{B}$ & HCV NS5b RNA Polymerase in complex inhibitor (5ee) & $580 / 580$ & $20 / 18$ \\
\hline $2 \mathrm{D} 3 \mathrm{U}$ & $\mathrm{A} / \mathrm{B}$ & X-ray crystal str of HCV RNA polymerase in complex & $570 / 570$ & $11 / 11$ \\
\hline 2D41 & $\mathrm{A} / \mathrm{B}$ & X-ray crystal str of HCV RNA dependent complex & $570 / 570$ & $12 / 12$ \\
\hline $2 \mathrm{GC} 8$ & $\mathrm{~A} / \mathrm{B}$ & Str. of HCV NS5b Polymerase & $578 / 578$ & $7 / 8$ \\
\hline $2 \mathrm{HWH}$ & $\mathrm{A} / \mathrm{B}$ & HCV NS5B allosteric inhibitor complex & $578 / 576$ & $14 / 14$ \\
\hline 2I1R & $\mathrm{A} / \mathrm{B}$ & Novel Thiazolones as HCV NS5B Polymerase & $576 / 576$ & $14 / 13$ \\
\hline${ }^{b} 1 \mathrm{RTL}$ & $\begin{array}{l}\mathrm{A} / \mathrm{B} \\
\mathrm{C} / \mathrm{D}\end{array}$ & Str. of HCV NS3 protease domain & $\begin{array}{r}200 / 200 \\
23 / 23\end{array}$ & $58 / 45$ \\
\hline${ }^{b} 1 \mathrm{NS} 3$ & $\begin{array}{l}\mathrm{A} / \mathrm{B} \\
\mathrm{C} / \mathrm{D}\end{array}$ & Str. of $\mathrm{HCV}$ protease (BK strain) & $\begin{array}{r}186 / 186 \\
14 / 14\end{array}$ & $64 / 66$ \\
\hline b $2 \mathrm{~A} 4 \mathrm{R}$ & $\begin{array}{l}\mathrm{A} / \mathrm{C} \\
\mathrm{B} / \mathrm{D}\end{array}$ & HCV NS3 Protease Domain & $\begin{array}{r}200 / 200 \\
23 / 23 \\
\end{array}$ & $58 / 84$ \\
\hline
\end{tabular}

b To compute the numbers of binding sites, we considered the 2 longest chains in each protein complex, for the reason that chains $\mathrm{C}$ and $\mathrm{D}$ of $1 \mathrm{~A} 1 \mathrm{R}, 1 \mathrm{RTL}, 1 \mathrm{NS} 3$, chain $\mathrm{P}$ of $1 \mathrm{N64}$, and chains $\mathrm{B}$ and $\mathrm{D}$ of $2 \mathrm{~A} 4 \mathrm{R}$ are very short sequences, and have limited influence on binding site identification.

This column gives the numbers of binding sites (identified by ASA method) in the 2 longest chains of HCV complexes.

Conformational parameters such as sequence entropy, residue binding propensity, evolutionary distance, secondary structure, as well as residue biological-physical properties, are coupled as input feature vectors for the RBFNN model for locating binding residues. The model was trained and tested using a dataset of HCV complexes with multiple chains, which were retrieved from the protein data bank (PDB) 3]. Simulation results indicate that our proposed method can successfully predict binding sites within HCV complexes.

\section{Materials}

We extracted $64 \mathrm{HCV}$ complexes with known structures from PDB, and removed the proteins with identity of $90 \%$ or higher using the program PSI-BLAST [4]. We then selected the protein complexes with multiple chains as the representative set of HCV protein complexes. 18 structurally non-redundant HCV complexes with multiple chains were left for our study of binding site prediction, as shown in Table 1.

The accessible surface area (ASA) method [5] was adopted to compute binding residues within the $\mathrm{HCV}$ complexes. Specifically, in a given $\mathrm{HCV}$ protein sequence, we calculated the ASA for each residue both in the unbound molecule 
Table 2. Binding residues in the HCV complexes

\begin{tabular}{|c|c|c|c|c|c|c|c|c|c|c|c|c|c|c|c|c|c|c|c|c|c|}
\hline \multirow{2}{*}{$\begin{array}{l}\mathrm{HCV} \\
\text { ID }\end{array}$} & \multicolumn{20}{|c|}{ Number of binding residue } & \multirow[b]{2}{*}{ SUM } \\
\hline & $\overline{\mathrm{A}}$ & $\overline{\mathrm{R}}$ & $\mathrm{N}$ & $\mathrm{D}$ & $\mathrm{C}$ & $\bar{Q}$ & $\mathrm{E}$ & $G$ & $\mathrm{H}$ & $\mathrm{T}$ & $\mathrm{L}$ & $\mathrm{K}$ & $\bar{M}$ & $\mathrm{~F}$ & $\mathrm{P}$ & $\mathrm{S}$ & $\mathrm{T}$ & $\bar{W}$ & $\bar{Y}$ & $\bar{V}$ & \\
\hline$\overline{1 \mathrm{CSJ}}$ & 4 & $\overline{4}$ & 4 & 4 & 2 & 2 & 6 & & 2 & 2 & 2 & 4 & & & 3 & 8 & 4 & & 4 & 2 & 65 \\
\hline $1 \mathrm{NHU}$ & & 6 & 2 & 4 & & 2 & 2 & & & & 4 & 2 & & & & & & & & 2 & 24 \\
\hline $1 \mathrm{~A} 1 \mathrm{R}$ & 8 & 8 & & 2 & 3 & 6 & 4 & 5 & 2 & 5 & 7 & & & 2 & 9 & 6 & 14 & 2 & 3 & 10 & 96 \\
\hline $1 \mathrm{C} 2 \mathrm{P}$ & & 6 & 2 & 6 & & 2 & 2 & & 2 & & 4 & 2 & & & & & & & & 2 & 28 \\
\hline 1HEI & 16 & 11 & 4 & 17 & 3 & 10 & 18 & 21 & 9 & 6 & 15 & 14 & 6 & 5 & 16 & 13 & 25 & 1 & 4 & 20 & 234 \\
\hline $1 \mathrm{~N} 64$ & 9 & 7 & 10 & 7 & 1 & 9 & 11 & 4 & & 3 & 7 & 18 & 2 & 6 & 14 & 26 & 19 & 4 & 8 & 8 & 173 \\
\hline $1 \mathrm{NHV}$ & & 6 & 2 & 4 & & 2 & 2 & & & & 4 & 2 & & & & & & & & 2 & 24 \\
\hline 1ZH1 & 5 & 3 & 2 & & 4 & 2 & 1 & & 2 & & & 2 & & 4 & 5 & 4 & 4 & 2 & 3 & & 43 \\
\hline $2 \mathrm{AWZ}$ & & 3 & & 2 & & 4 & 3 & 9 & & & 5 & 5 & 1 & 1 & 2 & 2 & & & & & 37 \\
\hline $2 \mathrm{AX} 1$ & 1 & 4 & & 2 & & 4 & 2 & 8 & & 1 & 5 & 5 & 1 & 1 & 2 & 2 & & & & & 38 \\
\hline $2 \mathrm{D} 3 \mathrm{U}$ & & 4 & 2 & 4 & & 2 & 2 & & & & 4 & 2 & & & & & & & & 2 & 22 \\
\hline $1 \mathrm{NS} 3$ & 11 & 10 & 1 & 6 & 5 & 11 & 4 & 10 & 2 & 4 & 13 & 5 & & 2 & 4 & 9 & 13 & 2 & 4 & 14 & 130 \\
\hline $2 \mathrm{D} 41$ & & 6 & 2 & 4 & & 2 & 2 & & & & 4 & 2 & & & & & & & & 2 & 24 \\
\hline $2 \mathrm{GC} 8$ & 2 & & & & & & & 1 & & & 2 & 6 & & & & 2 & 2 & & & & 15 \\
\hline $2 \mathrm{HWH}$ & & 5 & 2 & 6 & & 2 & 2 & & 2 & & 4 & 3 & & & & & & & & 2 & 28 \\
\hline 1RTL & 12 & 8 & & 4 & 3 & 6 & 4 & 5 & 2 & 6 & 7 & & & 2 & 11 & 5 & 13 & 2 & 3 & 10 & 103 \\
\hline $2 \mathrm{I} 1 \mathrm{R}$ & & 5 & 2 & 6 & & 2 & 2 & & 2 & & 4 & 2 & & & & & & & & 2 & 27 \\
\hline $2 \mathrm{~A} 4 \mathrm{R}$ & 13 & 14 & 2 & 9 & 3 & 9 & 6 & 8 & 2 & 6 & 7 & 3 & 2 & & 14 & 8 & 17 & 1 & 6 & 12 & 142 \\
\hline
\end{tabular}

and in the complex using the program GETAREA 1.1 6] with a probe sphere of radius $1.4 \AA$. An amino acid is defined to be a binding residue if its ASA in the complex is less than that in the monomer by at least $1 \AA^{2}$ [7. In this way, we computed the number of binding residues for each HCV complex (Table 2).

\section{Methods}

Due to the simple topological structure and powerful nonlinear mapping ability, the radial basis function neural network (RBFNN) model has been successfully adopted to solve nonlinear classification problems with multi-parameters and multi-models, including protein inter-residue contact map prediction [8, 2]. We adopted the RBFNN model, described in our previous study [2], to address the binding sites prediction problem.

\subsection{Sequence Entropy}

The importance of different residue for maintaining structure stability and particular function of a protein can usually be inferred from the alignment of that protein and its homologies. These information could provide some useful guidelines for analyzing and predicting HCV-human protein binding sites. In the present study, we used the normalized sequence entropy score [1] to measure the variability at a particular position of a given HCV protein sequence. Specifically, this sequence entropy is defined by the following equation:

$$
S_{\text {column }(j)}=-\sum_{k=1}^{20} P_{i} \ln P_{i} \times \frac{1}{\ln K}
$$




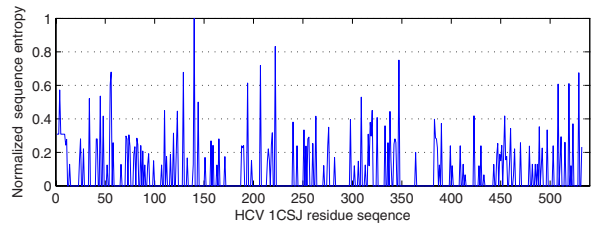

(a) Normalized sequence entropy

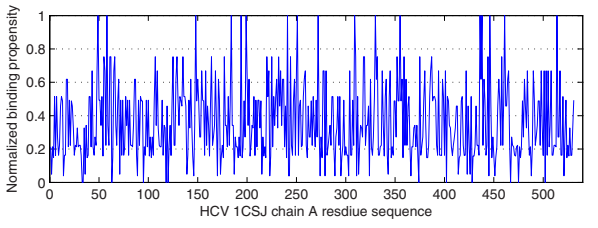

(b) Normalized residue binding propensity

Fig. 1. Distributions of sequence entropy and residue binding propensity of chain A of HCV protein 1CSJ. (a) Normalized sequence entropy of residues along chain A of 1CSJ, (b) Normalized residue propensity of chain A of 1CSJ.

where $P(i)=\frac{n_{i}}{N}$ is the fractional frequency of type $i$ residue in the $j$ th column, $N=\sum_{i=1}^{20}\left(n_{i}\right)$ is the total occurrences of all residues in the column. A low value of sequence entropy, $S_{\text {column }(j)}$, implies that position $j$ has been subjected to relatively higher evolutionary conservation than other positions in the same alignment having a higher sequence entropy.

Considering HCV protein 1CSJ as an example, its 14 homologous sequences (PDB ID: 2D3U, 2BRK, 1QUV, 1NHU, 2AWZ, 1OS5, 2I1R, 2HAI, 1GX5, 1GX6, $1 \mathrm{C} 2 \mathrm{P}, 1 \mathrm{NB} 4,1 \mathrm{YVE}$, and $1 \mathrm{YUY}$ ) were retrieved from the PDB by using the program BLASTP [12. Then, its sequence entropy was calculated from the alignment of these homologies by the help of Bioinformatics Toolbox of MATLAB 7.1, which is illustrated in Fig 1(a).

\subsection{Binding Propensity}

Several previous studies demonstrated that some critical amino acids, such as structural or functional conserved residues, contribute significantly to protein binding segments, as well as to protein-protein interaction [13,14, 15. This suggests that some particular residues may have higher propensities than others in protein-protein binding interactions, and these propensities can in some degree be used to improve binding site prediction. The frequencies of residues in the binding sites of HCV complexes can be used to derive the residue propensities. The overall propensities of the 20 amino acids were computed based on the occurrences of each residue, as shown in Table 2 Briefly, for a particular type residue $R_{i}$, the binding propensity $P_{b i n d}\left(R_{i}\right)$ is defined as:

$$
\begin{gathered}
P_{\text {bind }}\left(R_{i}\right)=\frac{f_{\text {bind }}^{R_{i}}}{f_{\text {whole }}^{R_{i}}} \\
f_{\text {bind }}^{R_{i}}=\frac{O_{\text {bind }}\left(R_{i}\right)}{\sum_{i=1}^{20} O_{\text {bind }}\left(R_{i}\right)} \\
f_{\text {whole }}^{R_{i}}=\frac{N\left(R_{i}\right)}{\sum_{i=1}^{20} N\left(R_{i}\right)}
\end{gathered}
$$


Table 3. Binding propensities of residue

\begin{tabular}{|c|c|c|c|c|c|}
\hline $\begin{array}{r}\text { Residue } \\
\text { Type } \\
\end{array}$ & $\begin{array}{l}\text { Num. of } \\
\text { Residues }\end{array}$ & $f_{\text {whole }}^{r_{i}}$ & $\begin{array}{r}\text { Num. of } \\
\text { Binding } \\
\text { Residues } \\
\end{array}$ & $f_{\text {bind }}^{r_{i}}$ & $\begin{array}{r}\text { Binding } \\
\text { Propensity } \\
P_{\text {bind }}\left(r_{i}\right) \\
\end{array}$ \\
\hline $\mathrm{A}$ & 1361 & 5.01 & 81 & 6.51 & 1.29 \\
\hline $\mathrm{R}$ & 945 & 3.49 & 110 & 8.84 & 2.53 \\
\hline $\mathrm{N}$ & 475 & 1.76 & 37 & 2.97 & 1.69 \\
\hline $\mathrm{D}$ & 691 & 2.55 & 87 & 6.99 & 2.74 \\
\hline $\mathrm{C}$ & 526 & 1.94 & 24 & 1.93 & 0.99 \\
\hline Q & 501 & 1.85 & 77 & 6.18 & 3.34 \\
\hline $\mathrm{E}$ & 659 & 2.43 & 73 & 5.86 & 2.41 \\
\hline G & 989 & 3.65 & 71 & 5.70 & 1.56 \\
\hline $\mathrm{H}$ & 656 & 2.42 & 27 & 2.17 & 0.89 \\
\hline I & 356 & 1.31 & 33 & 2.65 & 2.02 \\
\hline $\mathrm{L}$ & 1481 & 5.47 & 98 & 7.87 & 1.44 \\
\hline K & 797 & 2.94 & 77 & 6.18 & 2.10 \\
\hline M & 257 & 0.95 & 12 & 0.96 & 1.02 \\
\hline $\mathrm{F}$ & 390 & 1.44 & 23 & 1.85 & 1.28 \\
\hline $\mathrm{P}$ & 832 & 3.07 & 80 & 6.42 & 2.09 \\
\hline $\mathrm{S}$ & 1304 & 4.82 & 85 & 6.83 & 1.42 \\
\hline $\mathrm{T}$ & 1121 & 4.14 & 111 & 8.92 & 2.15 \\
\hline W & 236 & 0.87 & 14 & 1.12 & 1.29 \\
\hline $\mathrm{Y}$ & 605 & 2.24 & 35 & 2.81 & 1.26 \\
\hline $\mathrm{V}$ & 1130 & 4.18 & 90 & 7.22 & 1.73 \\
\hline SUM & 27065 & 100 & 1245 & 100 & \\
\hline
\end{tabular}

where $f_{\text {bind }}^{R_{i}}$ is the fraction of residue $R_{i}$ in the binding sites, $O_{b i n d}\left(R_{i}\right)$ is the number of observed occurrence of residue $R_{i}, N\left(R_{i}\right)$ denotes the number of $r_{i}$ residue in the whole $\mathrm{HCV}$ set. Residues with a higher propensity indicate that the residue occurs more frequently in the binding surface in comparison with other residues with lower propensity. Table 3 displays the detailed binding propensities of 20 different types of residue. For the purpose of encoding the binding propensity to the input feature vector, we normalized all the computed propensities. In this manner, all the 20 residues' propensities lie in the area of $[0,1]$. Detailed distribution of residue binding propensity along the chain $\mathrm{A}$ of HCV 1CSJ was demonstrated in Fig 1(b).

\subsection{Encoding Scheme}

Conformational features such as sequence entropy, binding propensity, residue type, residue classification, and secondary structure, were integrated to construct the input feature vector of our RBFNN model for each residue. Specifically, sequence entropy was represented by a 5-bit vector, i.e., 00001 denotes the sequence entropy which value lies in the region of $[0,0.2), 00010$ denotes the sequence entropy in the range of $[0.2,0.4), \cdots \cdots, 10000$ denotes the region of $[0.8,1]$. Furthermore, binding propensity is also represented by a 5 -bit vector, which is similar to that of sequence entropy. The detailed encoding scheme of the other three conformational attributes, residue type, residue classification and secondary structure, can be found in our previous study [2]. Hence, a given residue is represented by a 39 -bit $(5+5+20+6+3)$ vector. Moreover, when we attempt to determine whether a particular residue is in a binding site, the sliding windows method [16, 17, was adopted to construct an input feature vector of our RBFNN predictor, to consider the contribution of sequence adjacent residues. 


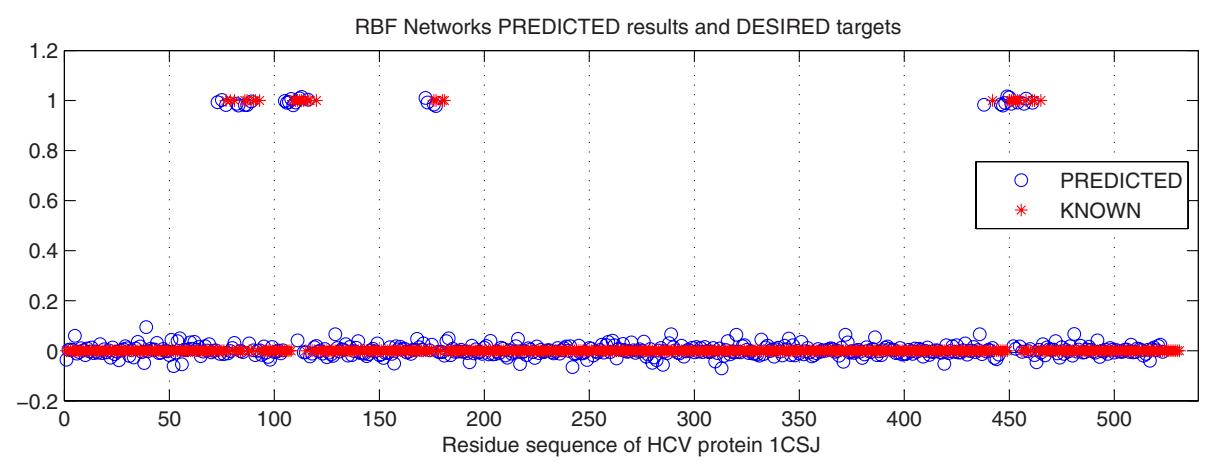

Fig. 2. Predicted and known targets of chain_A within HCV protein sequence 1CSJ, blue ' $O$ ' denotes the predicted binding sites, while red ' $*$ ' is the known binding sites

Table 4. Detailed predicted results of the proposed RBFNN model

\begin{tabular}{|c|c|c|c|c|c|c|}
\hline \multirow{2}{*}{$\begin{array}{l}\text { PDB } \\
\text { code }\end{array}$} & \multicolumn{2}{|c|}{$\begin{array}{c}\text { Correctly } \\
\text { predicted numbers }\end{array}$} & \multicolumn{2}{|c|}{$\begin{array}{c}\text { Incorrectly } \\
\text { predicted numbers }\end{array}$} & \multirow[b]{2}{*}{ Specificity } & \multirow[b]{2}{*}{ Sensitivity } \\
\hline & $\overline{T_{p}}$ & $F_{p}$ & $\overline{T_{n}}$ & $\overline{F_{n}}$ & & \\
\hline 1CSJ & 47 & 959 & 38 & 18 & $3.81 \%$ & $76.17 \%$ \\
\hline $1 \mathrm{NHU}$ & 18 & 1106 & 26 & 6 & $2.30 \%$ & $77.51 \%$ \\
\hline $2 \mathrm{AX} 1$ & 29 & 1104 & 18 & 9 & $1.60 \%$ & $78.03 \%$ \\
\hline Average & & & & & $3 \%$ & $77 \%$ \\
\hline
\end{tabular}

\section{Results and Discussion}

Here we take the HCV protein sequence, 1CSJ, as an example to illustrate the result. The predicted scores of 531 amino acid residues along the chain A of 1CSJ are shown in Fig 2 The predicted scores of most residues lie in the area of $[-0.15,0.15]$, and are labeled as non-binding sites. Roughly, the predicted binding residues have scores in the range of $[0.97,1.05]$, and all the predicted binding sites were located in the known 4 binding segments successfully. Fig 3 presents the predicted and known binding sites for HCV 1NHU chain A and 2AX1 chain A. The results indicate that our proposed method and RBFNN predictor can catch the binding sites successfully.

To evaluate the performance of the RBFNN model, 3 randomly selected HCV complexes, 1CSJ, 1NHU, and 2AX1, were selected as a test set. As shown in Table 4 , the sensitivity and specificity of the model are $77 \%$ and $3 \%$, respectively. Moreover, our RBFNN model successfully predicted 94 binding sites out of the 127 binding sites from the 3 testing protein complexes, with an overall prediction accuracy of $74 \%$. Although the sensitivity is relatively high, the specificity remains to be improved. The residue properties, which were not used in this study, such as positive charge, negative charge, polar, and hydrophobic, can be encoded 


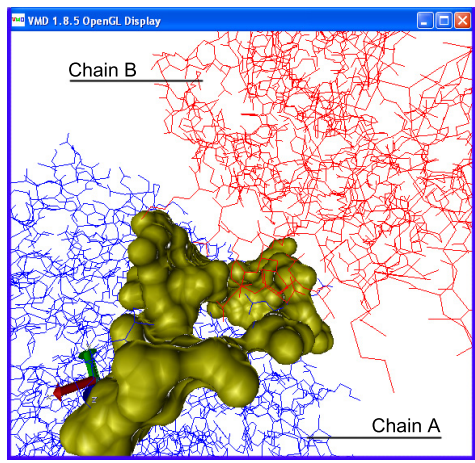

(a) Known binding sites of $1 \mathrm{NHU}$

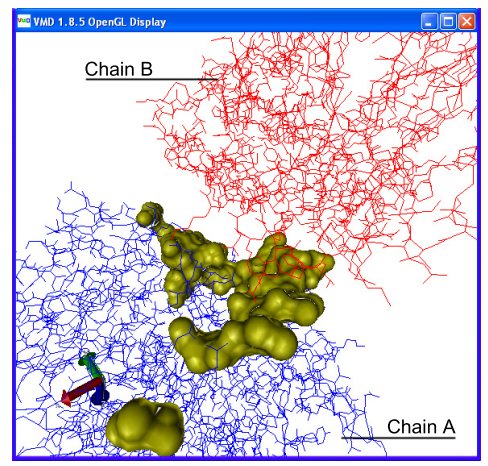

(b) Predicted biding sites of $1 \mathrm{NHU}$

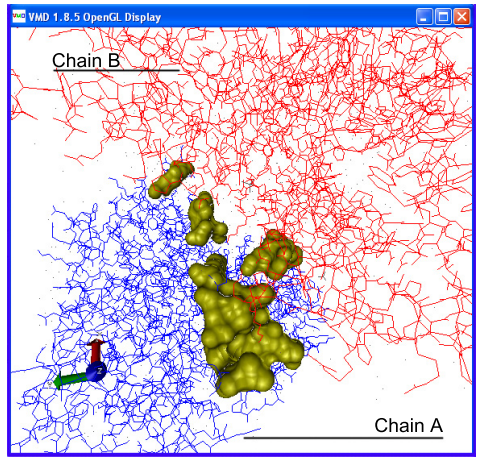

(c) Known binding sites of $2 \mathrm{AX} 1$

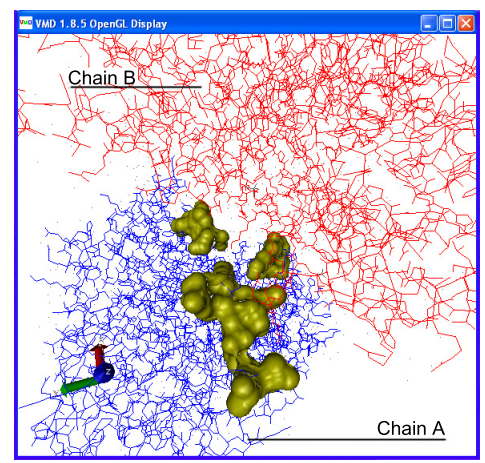

(d) Predicted binding sites of $2 \mathrm{AX} 1$

Fig. 3. Comparison of predicted results and known binding area within chain A of $\mathrm{HCV}$ proteins $1 \mathrm{NHU}$ and $2 \mathrm{AX} 1$

for more accurate prediction. However, prediction with accuracy $74 \%$ of binding sites only with residue binding propensity and sequence entropy indicates this method is very powerful and promising for narrowing down the search space.

\section{Conclusion}

We have developed a method for predicting binding sites in HCV complexes using the statistical residue conformational property (binding propensity) and protein evolutionary information (sequence entropy). Experimental results demonstrate that the method can accurately predict binding residues in HCV complexes. In our next work, we will focus on the problem of identifying interaction areas between HCV and human proteins, and the results of this study will help identify interaction segments between $\mathrm{HCV}$ and human proteins. 
Acknowledgments. This work was supported in part by the Korea Research Foundation Grant funded by the Korean Government (KRF-2006-D00038) and in part by KOSEF through the Systems Bio-Dynamics Research Center.

\section{References}

1. Drummer, H.E., Wilson, K.A. and Poumbourios, P.: Identification of the Hepatitis C Virus E2 Glycoprotein Binding Site on the Large Extracellular Loop of CD81. Journal of Virology, 76(21) (2002), 11143-11147

2. Zhang, G.Z., Huang, D.S.: Prediction of inter-residue contacts map based on genetic algorithm optimized radial basis function neural network and binary input encoding scheme. Journal of Computer Aided Molecular Design, 18 (2004), 797-810

3. Berman, H.M., Westbrook, J., Feng, Z. and et al.: The Protein Data Bank. Nucleic Acids Research, 28 (2000), 235-242.

4. Altschul, S.F., Madden, T.L., Schaffer, A.A. and et al.: Gapped BLAST and PSI-BLAST: a new generation of protein database search programs. Nucleic Acids Research, 25 (1997), 3389-3402

5. Re $\breve{s}$, I. and Lichtarge, O.; Character and evolution of protein-protein interfaces. Physical Biology, 2 (2005), S36-S43

6. Fraczkiewicz, R. and Braun, W.: Exact and efficient analytical calculation of the accessible surface areas and their gradients for macromolecules. Journal of Computational Chemistry, 19 (1998), 319-333

7. Valdar, W.S.J. and Thornton, J.M.: Conservation Helps to Identify Biologically Relevant Crystal Contacts. J. Mol. Biol., 313 (2001), 399-416

8. Fariselli, P., and Casadio, R.: Neural network based prediction of residue contacts in protein. Protein Engineering, 12 (1999), 15-21

9. Jores, R., Alzari, P.M, Meo, T.: Resolution of hypervariable regions in T-cell receptor $\beta$ chains by a modified Wu-Kabat index of amino acid diversity. Proc. Natl. Acad. Sci. USA, 87 (1990), 9138-9142

10. Schneider, T.D.: Information content of individual genetic sequences. Journal of Theoretical Biology, 189 (1997), 427-441

11. Guharoy, M. and Chakrabarti, P.: Conservation and relative importance of residues across protein-protein interfaces. Proc. Natl. Acad. Sci. USA, 102 (2005), $15447-15452$

12. Schäffer, A.A., Aravind, L., Madden, T.L., Shavirin, S. and et al.: Improving the accuracy of PSI-BLAST protein database searches with composition-based statistics and other refinements. Nucleic Acids Research, 29 (2001), 2994-3005.

13. Hu, Z.J., Ma, B.Y., Wolfson, H. and Nussinov, R.: Conservation of Polar Residues as Hot Spots at Protein Interfaces. PROTEINS: Structure, Function, and Genetics, 39 (2000), 331-342

14. Ma, B., Elkayam, T., Wolfson, H. and Nussinov, R.: Protein-protein interactions: Structurally conserved residues distinguish between binding sites and exposed protein surfaces. Proc. Natl. Acad. Sci. USA, 100(10) (2003), 5772-5777

15. De-Vries, S.J. and Bonvin, A.M.J.J.: Intramolecular surface contacts contain information about protein-protein interface regions. Bioinformatics, 22(17) (2006), 2094-2098

16. Rost, B. and Sander, C.: Prediction of Protein Secondary Structure at Better than 70\% Accuracy. J. Mol. Biol., 232(1) (1993), 584-599

17. Kim, K. and Park, H.: Protein secondary structure prediction based on an improved support vector machines approach. Protein Engineering, 16(8) (2003), 553-560 